\title{
RECYCLING GIVENS ROTATIONS FOR THE EFFICIENT APPROXIMATION OF PSEUDOSPECTRA OF BAND-DOMINATED OPERATORS
}

\author{
MARKO LINDNER AND TORGE SCHMIDT
}

\begin{abstract}
We study spectra and pseudospectra of certain bounded linear operators on $\ell^{2}(\mathbb{Z})$. The operators are generally non-normal, and their matrix representation has a characteristic offdiagonal decay. Based on a result of Chandler-Wilde, Chonchaiya and Lindner for tridiagonal infinite matrices, we demonstrate an efficient algorithm for the computation of upper and lower bounds on the pseudospectrum of operators that are merely norm limits of band matrices - the socalled band-dominated operators. After approximation by a band matrix and fixing a parameter $n \in \mathbb{N}$, one looks at $n$ consecutive columns $\{k+1, \ldots, k+n\}, k \in \mathbb{Z}$, of the corresponding matrix and computes the smallest singular value of that section via $\mathrm{QR}$ factorization. We here propose a QR factorization by a sequence of Givens rotations in such a way that a large part of the computation can be reused for the factorization of the next submatrix - when $k$ is replaced by $k+1$. The computational cost for the next factorization(s) is $\mathscr{O}(n d)$ as opposed to a naive implementation with $\mathscr{O}\left(n d^{2}\right)$, where $d$ is the bandwidth. So our algorithm pays off for large bands, which is attractive when approximating band-dominated operators with a full (i.e. not banded) matrix.
\end{abstract}

Mathematics subject classification (2010): Primary 65J10, secondary 47A10, 47B36, 65F15. Keywords and phrases: pseudospectra, Jacobi matrix, band-dominated operator, QR factorization.

\section{REFERENCES}

[1] G. R. Barrenechea, L. Boulton And N. Boussaid, Eigenvalue enclosures, arXiv:1306.5354.

[2] Z. Bai, J. Demmel, J. Dongarra, A. Ruhe and H. van der Vorst, Templates for the Solution of Algebraic Eigenvalue Problems - A Practical Guide, siam, first edition, 2000.

[3] M. Van Barel, R. Vandebril, P. Van Dooren and K. FrederiX, Implicit double shift qralgorithm for companion matrices, Numerische Mathematik, Springer, vol. 116 (2), p. 117-212.

[4] C. Bekas And E. Gallopoulos, Cobra: Parallel path following for computing the matrix pseudospectrum, Parallel Computing 27 (2001), 1879-1896.

[5] J. Ben-Artzi, A. C. Hansen, O. Nevanlinna And M. Seidel, New barriers in complexity theory: On the Solvability Complexity Index and towers of algorithms, C. R. Acad. Sci. Paris, Ser. I 353 (2015), 931-936.

[6] A. BÖTTCHER, M. EMBREE AND M. LINDNER, Spectral approximation of banded Laurent matrices with localized random perturbations, Integr. Equ. Oper. Theory 42 (2002), 142-165.

[7] A. Böttcher, M. Embree And V. I. SoKolov, Infinite Toeplitz and Laurent matrices with localized impurities, Linear Algebra Appl. 343/344 (2002), 101-118.

[8] A. Böttcher And B. Silbermann, Introduction to Large Truncated Toeplitz Matrices, Springer, Berlin, Heidelberg, 1999.

[9] N. Bosner, Fast Methods for Large Scale Singular Value Decomposition, Doctoral Thesis, University of Zagreb, 2006.

[10] M. BRUEHL, A curve tracing algorithm for computing the pseudospectrum, BIT 36 (3) (1996), 441454. 
[11] S. N. Chandler-Wilde, R. Chonchaiya And M. Lindner, Upper bounds on the spectra and pseudospectra of Jacobi and related operators, in preparation.

[12] S. N. Chandler-Wilde And M. Lindner, Coburn's Lemma and the Finite Section Method for Random Jacobi Operators, Journal of Functional Analysis, 270 (2016), 802-841.

[13] R. Chonchaiya, Computing the Spectra and Pseudospectra of Non-Self-Adjoint, Random Operators Arising in Mathematical Physics, PhD Thesis, University of Reading, UK, 2010, http://www.tuhh.de/ matmli/files/ChonchaiyaThesis.pdf.

[14] E. B. Davies And M. Plum, Spectral pollution, IMA J. Numer. Anal., 24 (2004), 417-438.

[15] J. GlobeVniK, Norm-constant analytic functions and equivalent norms, Illinois J. Math. 20 (1976), $503-506$.

[16] G. H. Golub And C. F. Van Loan, Matrix Computations, The Johns Hopkins University Press, first edition, 1983.

[17] R. Hagger, M. Lindner And M. SEIDEL, Essential pseudospectra and essential norms of banddominated operators, Journal of Mathematical Analysis and Applications, 437 (2016), 255-291.

[18] S. HAMmARLING, C. LUCAS, Updating the QR factorization and the least squares problem, Manchester Institute for Mathematical Sciences, MIMS EPrint: 2008.111 (2008).

[19] A. C. HANSEN, On the solvability complexity index, the n-pseudospectrum and approximations of spectra of operators, J. Amer. Math. Soc., 24 (2011), 81-124.

[20] V. G. Kurbatov, Functional Differential Operators and Equations, Kluwer Academic Publishers, Dordrecht, Boston, London, 1999.

[21] M. Lindner, Infinite Matrices and their Finite Sections: An Introduction to the Limit Operator Method, Frontiers in Mathematics, Birkhäuser 2006.

[22] M. Lindner, Fredholm Theory and Stable Approximation of Band Operators and Their Generalisations, Habilitation thesis, TU Chemnitz, Germany, 2009.

[23] M. LINDNER AND M. SEIDEL, An affirmative answer to a core issue on limit operators, Journal of Functional Analysis, 267 (2014), 901-917.

[24] D. Mezher And B. PhilipPe, PAT - a Reliable Path-Following Algorithm, Numerical Algorithms 29 (2002), 131-152.

[25] V. S. Rabinovich, S. Roch and B. Silbermann, Limit Operators and Their Applications in Operator Theory, Birkhäuser, 2004.

[26] L. Reichel AND L. N. TREFEThen, Eigenvalues and pseudo-eigenvalues of Toeplitz matrices, Lin. Alg. Appl. 162-164 (1992), 153-185.

[27] M. SEIDEL, On Some Banach Algebra Techniques in Operator Theory, PhD Thesis, TU Chemnitz, 2011.

[28] M. SEIDEL, On $(N, \varepsilon)$-pseudospectra of operators on Banach spaces, Journal of Functional Analysis, 262 (2012), 4916-4927.

[29] M. Seidel and B. Silbermann, Finite Sections of Band-Dominated Operators - Norms, Condition Numbers and Pseudospectra, Operator Theory: Advances and Applications, 228 (2013), 375-390.

[30] L. N. TRefethen And M. Embree, Spectra and Pseudospectra: the Behavior of Nonnormal Matrices and Operators, Princeton University Press, Princeton, NJ, 2005.

[31] R. Vandebril, M. Van Barel and N. Mastronard, Matrix Computations and semiseparable matrices, Md. Johns Hopkins Univ. Press, volume 1, 2008.

[32] R. Vandebril, M. VAn Barel and N. Mastronardi, Rotational Figures Latex Package, http://people.cs.kuleuven.be/ raf.vandebril/homepage/software/latex.php, 2010.

[33] T. G. WRight AND L. N. TREFETHEN, Pseudospectra of rectangular matrices, IMA Journal of Numerical Analysis 22 (2002), 501-519. 